\title{
Educational attainment could be a protective factor against obstructive sleep apnea: a study based on Mendelian randomization
}

\author{
Lijuan Song ${ }^{1,2 \#}$, Hao Li ${ }^{2 \#}$, Jia Wang ${ }^{2 \#}$, Junyang $\mathrm{Xie}^{2}$, Gui Chen ${ }^{2}$, Tianhao Liang ${ }^{2}$, Yiyan Wang ${ }^{2}$, Liyao Ye ${ }^{2}$, \\ Xiaofen Wang ${ }^{2}$, Xiaoxuan Kuang ${ }^{2}$, Mei Ren ${ }^{2}$, Jinfeng $\mathrm{Ye}^{2}$, Yaqian Tang ${ }^{2}$, Ketong $\mathrm{Ji}^{2}$, Wenjing Liao ${ }^{2}$, \\ Xiaowen Zhang ${ }^{2,3}$
}

${ }^{1}$ Department of Otolaryngology-Head and Neck Surgery, First Affiliated Hospital, Jinan University, Guangzhou, China; ${ }^{2}$ State Key Laboratory of Respiratory Disease, Department of Otolaryngology-Head and Neck Surgery, First Affiliated Hospital, Guangzhou Medical University, Guangzhou, China; ${ }^{3}$ Department of Otolaryngology-Head and Neck Surgery, Fifth Affiliated Hospital, Guangzhou Medical University, Guangzhou, China

Contributions: (I) Conception and design: All authors; (II) Administrative support: All authors; (III) Provision of study materials or patients: All authors; (IV) Collection and assembly of data: All authors; (V) Data analysis and interpretation: All authors; (VI) Manuscript writing: All authors; (VII) Final approval of manuscript: All authors.

\#These authors contributed equally to this work.

Correspondence to: Wenjing Liao; Xiaowen Zhang. State Key Laboratory of Respiratory Disease, Department of Otolaryngology-Head and Neck Surgery, The First Affiliated Hospital, Guangzhou Medical University, 151 Yanjiang Road, Guangzhou 510120, China.

Email: entxiaowen@163.com; entwenjing@163.com.

Background: Causality between education and obstructive sleep apnea (OSA) is not known.

Methods: Genetic variants, as instrumental variables for years of education, were derived from the Social Science Genetic Association Consortium. The outcome datasets related to OSA were from the FinnGen research project (www.finngen.fi/en/). Inverse variance-weighted, weighted-median, and Mendelian randomization-Egger analysis were used to estimate causal effects. To assess the robustness and horizontal pleiotropy of significant results, leave-one-out sensitivity analysis and Mendelian randomization-Egger regression analysis were conducted. The inverse variance-weighted method was undertaken to estimate the association between years of education and other known risk factors for OSA. Analyses were conducted using the Two Sample Mendelian Randomization package of R 4.0.3.

Results: Genetic predisposition towards 4.2 years of additional education was associated with a $27.8 \%$ lower risk of OSA [odds ratio $(\mathrm{OR})=0.722,95 \%$ confidence interval $(\mathrm{CI})$ : 0.566-0.921; $\mathrm{P}=0.009$ ]. Sensitivity analyses were consistent with a causal interpretation in which a major bias from genetic pleiotropy was unlikely. The Mendelian randomization assumptions did not seem to be violated. Genetic predisposition towards longer education was associated with a lower body mass index, fewer cigarettes smoked per day, and greater alcohol intake per week.

Conclusions: Our data indicated that education could be a protective factor against OSA. Potential mechanisms could include body mass index, tobacco smoking, and alcohol intake.

Keywords: Education; sleep apnea; mendelian randomization; causality

Submitted Nov 15, 2021. Accepted for publication Jan 17, 2022.

doi: $10.21037 /$ jtd-21-945

View this article at: https://dx.doi.org/10.21037/jtd-21-945 


\section{Introduction}

Obstructive sleep apnea (OSA) is characterized by recurrent episodes of partial or complete collapse of the upper airway during sleep, which results in intermittent hypoxia and excessive sleepiness. It affects $17 \%$ of women and $34 \%$ of men in the USA, and has a similar prevalence in other countries (1).

Synchronization of molecular, metabolic, and cardiovascular circadian oscillations is fundamental to human health. OSA disrupts such temporal congruence, and elicits hemodynamic, autonomic, chemical, and inflammatory disturbances with acute and long-term consequences for heart, brain, and circulatory and metabolic functions $(2,3)$. OSA etiology, however, is poorly understood (3). Hence, it is necessary to identify potential causal risk factors that will contribute to guiding prevention efforts.

A study of 3007 South Australians aged 15 years suggested that a high level of educational attainment (EA) was associated with a lower risk of OSA (relative risk) (3). Causal conclusions are rarely justified following metaanalysis methods, even with strong evidence from clinical observations. Moreover, a poor level of EA is an important risk factor of obesity, coronary heart disease, and type-2 diabetes mellitus, which are closely related to OSA $(4,5)$. However, the potential pathways involved in the association from EA to OSA have not been studied.

Mendelian randomization (MR) analysis is a novel study design based on genetic epidemiology. It can be employed to assess potentially causal relationships using genetic variants as instrumental variables (IVs) to explore the effect of modifiable exposures (e.g., education) on outcomes (e.g., OSA) (6). Those genetic variants are allocated randomly before birth and fixed upon conception, so a bias due to confounding and reverse causation is likely to be avoided (7).

Genome-wide associations studies (GWAS) look for associations between variations in DNA sequences and phenotypes of interest by studying individuals with different phenotypes (e.g., affected or not affected by a common complex disease) and determining their genotype at the positions of hundreds of thousands of single nucleotide polymorphisms (SNPs). More summary-level data from large GWAS consortia have become readily available in the public domain. We can implement MR analyses based on GWAS data, whereby gene-exposure measures are taken from one GWAS and gene-outcome measures are taken from another GWAS, which is known as "two-sample MR analysis".
We applied two-sample MR analysis to identify the potential causal association between EA and OSA risk. We present the following article in accordance with the STROBE reporting checklist (available at https://jtd. amegroups.com/article/view/10.21037/jtd-21-945/rc).

\section{Methods}

\section{Genetic variants associated with $E A$}

Lee et al. (8) reported a large meta-analysis of GWAS for EA. The latter was measured as the number of years of schooling completed in 766,345 people of European descent. In total, 1,271 independent [linkage disequilibrium (LD), $\left.\mathrm{r}^{2}<0.1\right]$ SNPs were identified to be associated robustly with EA in the Social Science Genetic Association Consortium at a GWAS threshold of $\mathrm{P}<5 \times 10^{-8}$. These SNPs explained $12 \%$ of the variation in EA across individuals (8). The F statistic of that study was 82 (much larger than the conventional value of 10), which suggested that the instruments used could predict EA (9). In addition, we undertook strict LD-clumping restricted to $\mathrm{r}^{2}<0.001$, clumping distance $>10,000 \mathrm{~kb}$, and retained SNPs at a GWAS threshold of $\mathrm{P}<5 \times 10^{-8}$ to eliminate $\mathrm{LD}$ and, finally, 310 EA-related SNPs were obtained. available online: https://cdn.amegroups.cn/static/public/jtd-21-945-01.xlsx summarizes the SNPs associated with EA.

\section{GWAS summary data on OSA}

The GWAS summary data for OSA, determined by International Statistical Classification of Diseases and Related Health Problems 10th Revision, were obtained from the FinnGen research project (www.finngen.fi/en/). A total of 5,263 patients with OSA and 83,167 controls were included. All participants were of European ancestry. Then, 237 SNPs were selected as IVs for MR analysis after removing the SNPs which lacked the required information, were palindromic, or had incompatible alleles.

\section{GWAS summary data for the risk factors for OSA}

We wished to investigate the potential mechanisms from education to OSA. We employed an inverse variance weighted (IVW) method to assess the potential causal relationship between EA and other common risk factors for OSA. Well-known risk factors include body mass index (BMI), tobacco smoking, and alcohol consumption (4). GWAS summary data for these phenotypes were extracted 
Table 1 Details of datasets included in analyses.

\begin{tabular}{lclll}
\hline Trait & Sample size & Consortium & Reference \\
\hline Years of schooling & 766,345 & The Social Science Genetic Association Consortium (SSGAC) & Lee et al. (8) \\
Sleep apnoea & 88,430 & FinnGen research project website & https://www.finngen.fi/en & Yengo et al. (10) \\
Body mass index & 681,275 & A meta-analysis of GWAS & Liu et al. (11) \\
Cigarettes per Day & 337,334 & GWAS and Sequencing Consortium of Alcohol and Nicotine use & Liu et al. (11) \\
Alcoholic drinks per week & 335,394 & GWAS and Sequencing Consortium of Alcohol and Nicotine use & &
\end{tabular}

GWAS, genome-wide association studies.

Table 2 Mendelian randomization estimates of the causality between EA and sleep apnoea

\begin{tabular}{lccccc}
\hline Exposure & Outcome & nSNPs & Method & OR $(95 \% \mathrm{Cl})$ & $\mathrm{P}$ \\
\hline Years of schooling & Sleep apnoea & 237 & IVW & $0.722(0.566-0.921)$ & 0.009 \\
& & & MR-Egger & $0.627(0.243-1.623)$ & 0.337 \\
& & Weighted median & $0.701(0.499-0.985)$ & 0.041 \\
\hline
\end{tabular}

EA, educational attainment; SNP, single-nucleotide polymorphism; OR, odds ratio; Cl, confidence interval; IVW, inverse variance weighted.

from GWAS and the Sequencing Consortium of Alcohol and Nicotine Use, and a meta-analysis of GWAS. Details of the risk factor-related GWAS included in MR analysis are given in Table $1(10,11)$.

\section{Statistical analyses}

We used several MR approaches to determine MR estimates of EA for OSA. We conducted a random-effects IVWbased meta-analysis of the Wald ratio for individual SNPs. We also estimated the effects using other statistical tests (weighted median and MR-Egger regression). The results are presented as odds ratios (OR) and $95 \%$ confidence intervals (CIs), which provided an estimate of relative risk caused by each standard deviation (SD) (4.2 years) increase in the years of schooling. Power calculations on MR analysis were carried out according to the method described by Brion and colleagues (12) if this value was greater than the conventional value of $80 \%$. Hence, the sample provided sufficient statistical power to detect a causal effect of EA upon OSA.

Moreover, MR analysis is relevant only if it employs IVs that are: (I) associated with the exposure; (II) independent of factors confounding the association of the exposure and outcome; (III) associated with the outcome only through exposure (13). To assess potential violation of these assumptions, we evaluated directional pleiotropy based on the intercept obtained from the MR-Egger analysis (14). We also carried out a leave-one-out sensitivity analysis in which we sequentially omitted one SNP at a time to evaluate if the MR estimate was driven or biased by a single SNP. All analyses were conducted in R 4.0.3 (R Institute for Statistical Computing, Vienna, Austria) using the Two Sample Mendelian Randomization package (15).

\section{Ethical statement}

The study was conducted in accordance with the Declaration of Helsinki (as revised in 2013). Patients were not involved in the design, recruitment, or conduct of the study. Hence, ethical approval was not needed for our study.

\section{Results}

\section{Causal effect from EA to OSA}

Genetically predicted higher EA was associated with a significantly lower odd of OSA (Table 2). Using conventional MR analysis, one SD-longer education (4.2 years) was associated with a $27.8 \%$ lower risk of OSA $(\mathrm{OR}=0.722$; 95\% CI: 0.566-0.921; $\mathrm{P}=0.009$ ) by the IVW method. Similar results were obtained using the weighted-median method. Although MR-Egger analysis failed to determine an association, the direction of the association was consistent and the OR was similar (forest plot in Figure S1). 
Table 3 Power for conventional Mendelian randomization analysis (two-sided $\alpha=0.05$ )

\begin{tabular}{|c|c|c|c|c|c|c|}
\hline Exposure & $\begin{array}{c}\text { R-squared } \\
\text { (of variance in EA } \\
\text { phenotype) }\end{array}$ & $\begin{array}{l}\text { Actual n } \\
\text { (FinnGen) }\end{array}$ & $\begin{array}{l}\text { Proportion of cases } \\
\text { (FinnGen) }\end{array}$ & $\begin{array}{c}\text { Observational } \\
\text { OR }\end{array}$ & $\begin{array}{l}\text { No. of required for } \\
80 \% \text { power }\end{array}$ & $\begin{array}{l}\text { Power at } \\
\text { actual } n\end{array}$ \\
\hline
\end{tabular}

EA, educational attainment (measured as number of years of schooling completed); OR, odds ratio.

Table 4 Causal effects from 4.2 years of education to common risk factors by IVW method

\begin{tabular}{lccc}
\hline Risk factors & nSNPs & OR (95\% Cl) & P \\
\hline Body mass index & 237 & $0.762(0.720-0.807)$ & $<0.001$ \\
Cigarettes per day & 294 & $0.702(0.650-0.758)$ & $<0.001$ \\
Alcoholic drinks per week & 293 & $1.042(1.011-1.074)$ & 0.008 \\
\hline
\end{tabular}

IVW, inverse variance weighted; SNP, single-nucleotide polymorphism; OR, odds ratio; Cl, confidence interval.

In addition, power calculations were based on a sample size of 88,430 (5,263 OSA cases and 83,167 controls) in the outcome datasets and a prevalence of type- 1 error of 0.05 . The number required for $80 \%$ power was $\geq 14,563$ participants. With a sample size of 88,430 , the outcome datasets provided sufficient statistical power $(>80 \%)$ to detect a causal effect of EA upon OSA (Table 3).

MR-Egger regression analysis did not reveal significant interception (intercept $\beta=0.002 ; \mathrm{SE}=0.007 ; \mathrm{P}=0.764$ ) and the funnel plot (Figure S2) was symmetrical, which indicated no evidence supporting the existence of pleiotropy. Regardless of SNP removal, there was no fundamental impact upon OSA (all lines were on the left side of 0 ) in the leave-one-out sensitivity analysis, which meant that the MR result was robust (Figure S3).

\section{Causal effect from $E A$ on the potential risk factors of OSA}

To identify risk factors that could mediate the association between EA and OSA, we investigated if higher EA was associated with several potential risk factors for OSA. Table 4 shows that 4.2 years-longer education years was associated with lower odds of body mass index $(\mathrm{OR}=0.762$, 95\% CI: $0.720-0.807 ; \mathrm{P}<0.001)$, lower odds of cigarettes per day $(\mathrm{OR}=0.702,95 \% \mathrm{CI}: 0.650-0.758 ; \mathrm{P}<0.001)$ and higher odds of alcoholic drinks per week ( $\mathrm{OR}=1.042$, 95\% CI: 1.011-1.074; $\mathrm{P}=0.008$ ).

\section{Discussion}

Using an MR study design, we found that higher EA was strongly associated with a lower risk of OSA, whereby 4.2 years of additional education predicted a reduction in OSA risk of $27.8 \%$. These results demonstrated the causeand-effect relationship between EA and OSA.

A few observational studies focusing on the relationship between education and OSA have been conducted (3), but their conclusions are not reliable for various reasons: small cohorts, measurement error, and methodical limitations. Our results may offer some of the strongest evidence to evaluate the causal role of EA in OSA because the questions mentioned before can be partly or completely avoided through a two-sample MR approach. By selecting summary statistics from the largest GWAS studies for education $(\mathrm{n}=766,345)$ and OSA (up to $\mathrm{n}=5,263$ cases and 83,167 controls), our data have enhanced statistical reliability.

Our results can denote the lifetime risk of OSA due to education because of the stability of genetic variants $(9,12-14)$. It has been reported that there may be a negative causal effect of education on BMI ( $\beta=-0.84 ; 95 \%$ CI: $-1.77-0.09)$ (16), which suggests that education could be a protective factor against obesity. Given that obesity is an established cause of OSA and is clearly related to education $(1,5,16)$, it may be a key intermediate factor on the education-OSA pathway. Inhalation of the contents of cigarette smoke causes irritation to the throat and subsequent local edema of the mucosa of the upper airways, which may be one of the contributing factors to upperairway obstruction and an increased risk of OSA (17). We showed that 4.2 years-longer education years was associated with $29.8 \%$ lower odds of cigarettes per day, which might be the reason why education could reduce OSA risk. 
Alcohol consumption has been shown to increase nasal and pharyngeal resistance acutely, and may be detrimental to sleep, affect sleep stages, and worsen insomnia and breathing disturbances during sleep (4). However, we showed that 4.2 years-longer education years was associated with $4.2 \%$ higher odds of alcoholic drinks per week. This finding appears to be contrary to the conclusion that education is a protective factor against OSA. However, this result focused only on the amount of alcohol consumed and ignored the type of alcohol consumed, which shows different effects on the human body. People with higher EA prefer the intake of more total alcohol (especially red wine), and less beer/cider and spirits, more frequent drinking, and consuming alcohol with meals (18), which may explain our findings. All those studies (including our study) do not specifically contribute to the debate on the mechanisms by which education impacts OSA.

This was the first MR study to investigate the causality between EA and OSA. The MR design can improve understanding of the causal effect of EA on OSA by reducing reverse causation and a potential bias from confounding. Our study had two main limitations. First, the MR analysis needs to be validated in more datasets related to OSA. Second, all GWAS data were from European populations, and whether our findings can be generalized to other populations is not known.

\section{Conclusions}

Our MR study supplied reliable evidence to demonstrate that education could be a protective factor against OSA. Future investigation is needed to elucidate the potential mechanisms that mediate the association between education and OSA.

\section{Acknowledgments}

We gratefully acknowledge the Social Science Genetic Association Consortium for access to their data. We are very thankful to the participants and investigators of the FinnGen study. Without their database, we could not have carried out this study.

Funding: This work was supported by China National Natural Science Foundation (No. 81900927).

\section{Footnote}

Provenance and Peer Review: This article was a standard submission to the Sleep Section in the journal. The article has undergone external peer review.

Reporting Checklist: The authors have completed the STROBE reporting checklist. Available at https://jtd. amegroups.com/article/view/10.21037/jtd-21-945/rc

Conflicts of Interest: All authors have completed the ICMJE uniform disclosure form (available at https://jtd.amegroups. com/article/view/10.21037/jtd-21-945/coif). The authors have no conflicts of interest to declare.

Ethical Statement: The authors are accountable for all aspects of the work in ensuring that questions related to the accuracy or integrity of any part of the work are appropriately investigated and resolved. The study was conducted in accordance with the Declaration of Helsinki (as revised in 2013). No patients were involved in the design, recruitment, or conduct of this study, so ethical approval was not needed.

Open Access Statement: This is an Open Access article distributed in accordance with the Creative Commons Attribution-NonCommercial-NoDerivs 4.0 International License (CC BY-NC-ND 4.0), which permits the noncommercial replication and distribution of the article with the strict proviso that no changes or edits are made and the original work is properly cited (including links to both the formal publication through the relevant DOI and the license). See: https://creativecommons.org/licenses/by-nc-nd/4.0/.

\section{References}

1. Gottlieb DJ, Punjabi NM. Diagnosis and Management of Obstructive Sleep Apnea: A Review. JAMA 2020;323:1389-400.

2. Floras JS. Sleep Apnea and Cardiovascular Disease: An Enigmatic Risk Factor. Circ Res 2018;122:1741-64.

3. Adams RJ, Piantadosi C, Appleton SL, et al. Investigating obstructive sleep apnoea: will the health system have the capacity to cope? A population study. Aust Health Rev 2012;36:424-9.

4. Pan Y, Wang W, Wang KS. Associations of Alcohol Consumption and Chronic Diseases With Sleep Apnea Among US Adults. Int J High Risk Behav Addict 2014;3:e19088.

5. McMartin A, Conley D. Commentary: Mendelian randomization and education-Challenges remain. Int J 
Epidemiol 2020;49:1193-206.

6. Smith GD, Ebrahim S. 'Mendelian randomization': can genetic epidemiology contribute to understanding environmental determinants of disease? Int J Epidemiol 2003;32:1-22.

7. Smith GD, Ebrahim S. Mendelian randomization: prospects, potentials, and limitations. Int J Epidemiol 2004;33:30-42.

8. Lee JJ, Wedow R, Okbay A, et al. Gene discovery and polygenic prediction from a genome-wide association study of educational attainment in 1.1 million individuals. Nat Genet 2018;50:1112-21.

9. Burgess S, Thompson SG; CRP CHD Genetics Collaboration. Avoiding bias from weak instruments in Mendelian randomization studies. Int J Epidemiol 2011;40:755-64.

10. Yengo L, Sidorenko J, Kemper KE, et al. Meta-analysis of genome-wide association studies for height and body mass index in 700000 individuals of European ancestry. Hum Mol Genet 2018;27:3641-9.

11. Liu M, Jiang Y, Wedow R, et al. Association studies of up to 1.2 million individuals yield new insights into the genetic etiology of tobacco and alcohol use. Nat Genet

Cite this article as: Song L, Li H, Wang J, Xie J, Chen G, Liang T, Wang Y, Ye L, Wang X, Kuang X, Ren M, Ye J, Tang Y, Ji K, Liao W, Zhang X. Educational attainment could be a protective factor against obstructive sleep apnea: a study based on Mendelian randomization. J Thorac Dis 2022;14(1):210215. doi: $10.21037 /$ jtd-21-945
2019;51:237-44.

12. Brion MJ, Shakhbazov K, Visscher PM. Calculating statistical power in Mendelian randomization studies. Int J Epidemiol 2013;42:1497-501.

13. Davey Smith G, Hemani G. Mendelian randomization: genetic anchors for causal inference in epidemiological studies. Hum Mol Genet 2014;23:R89-98.

14. Bowden J, Davey Smith G, Burgess S. Mendelian randomization with invalid instruments: effect estimation and bias detection through Egger regression. Int J Epidemiol 2015;44:512-25.

15. Hemani G, Zheng J, Elsworth B, et al. The MR-Base platform supports systematic causal inference across the human phenome. Elife 2018;7:34408.

16. Böckerman P, Viinikainen J, Pulkki-Råback L, et al. Does higher education protect against obesity? Evidence using Mendelian randomization. Prev Med 2017;101:195-8.

17. Patel SR. Obstructive Sleep Apnea. Ann Intern Med 2019;171:ITC81-96.

18. Zhou T, Sun D, Li X, et al. Educational attainment and drinking behaviors: Mendelian randomization study in UK Biobank. Mol Psychiatry 2021;26:4355-66. 


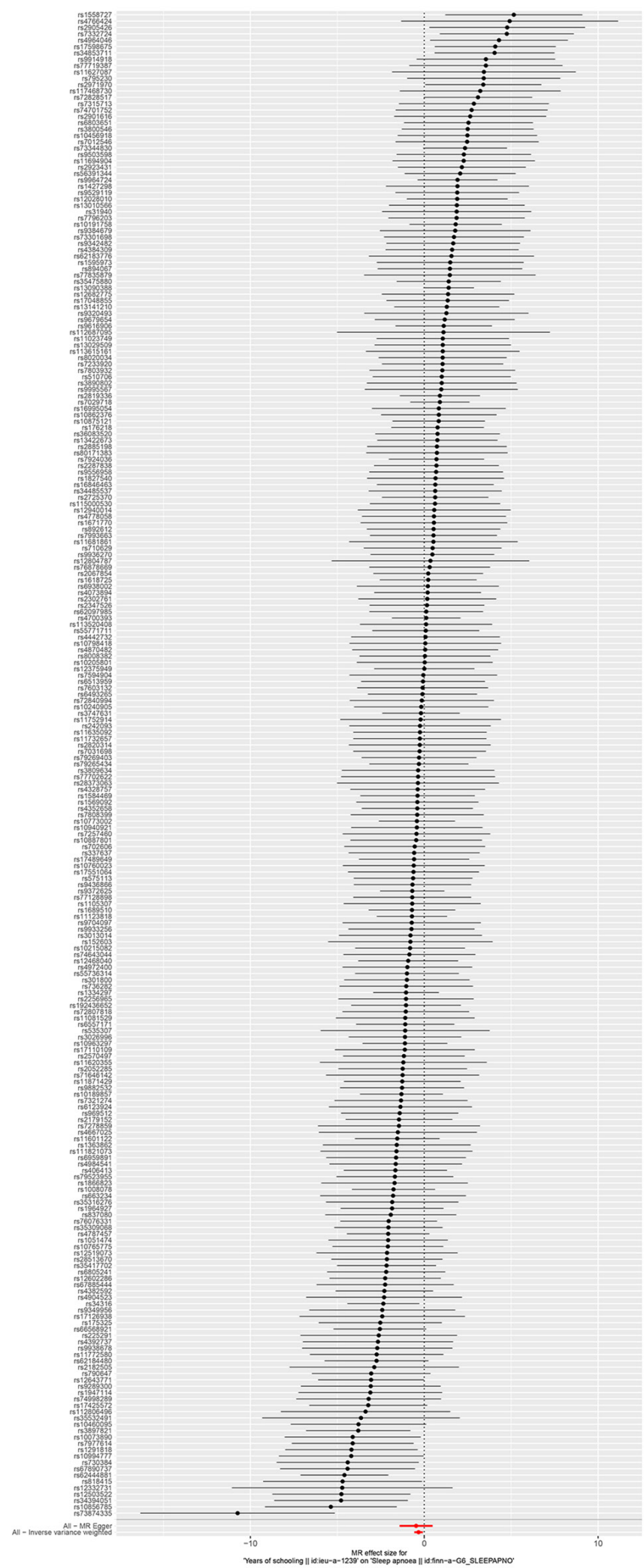

Figure S1 Forest plot of single nucleotide polymorphisms (SNPs) associated with educational attainment and their risk of sleep apnoea. 


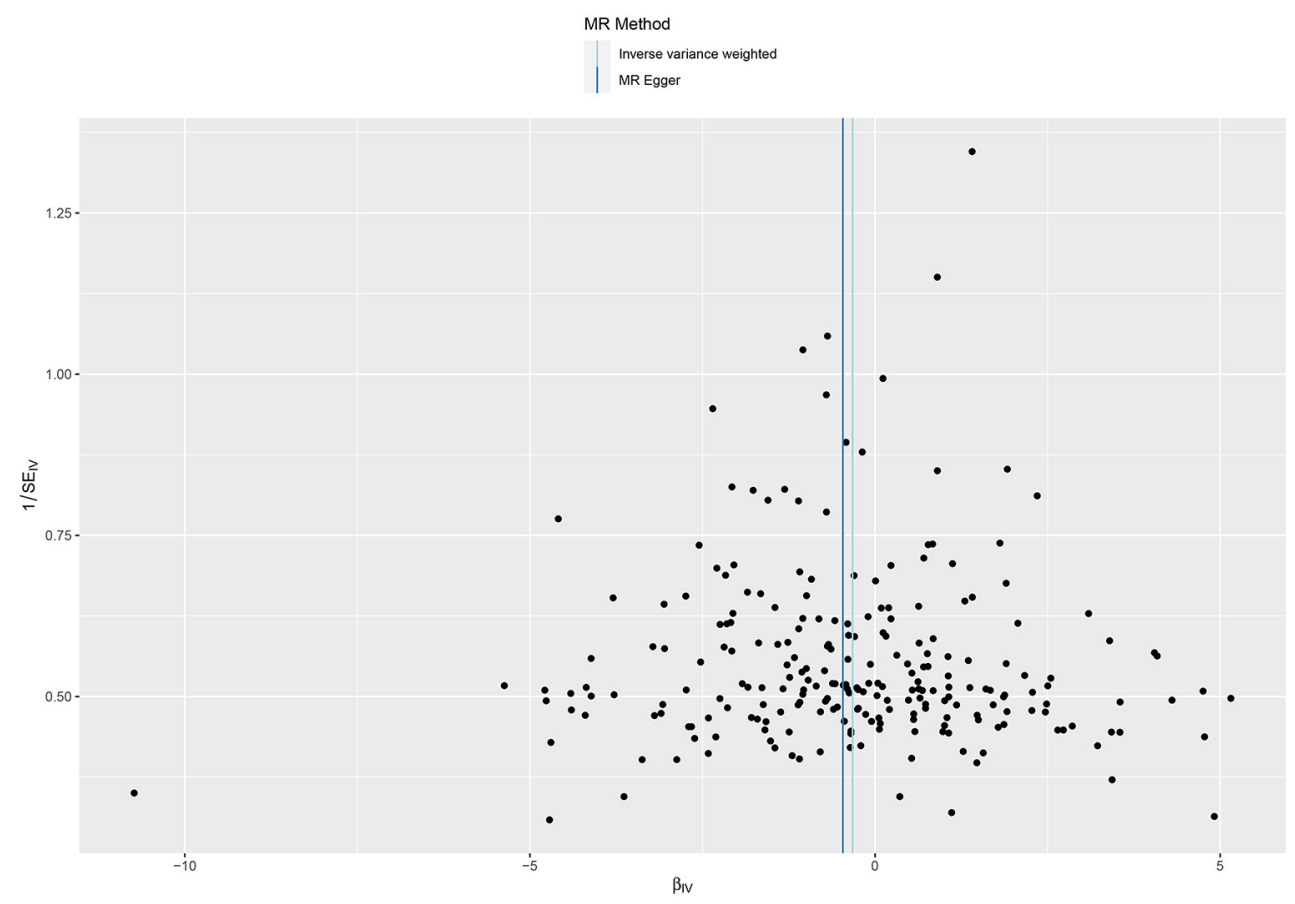

Figure S2 Funnel plot of single nucleotide polymorphisms (SNPs) associated with educational attainment and their risk of sleep apnoea. 


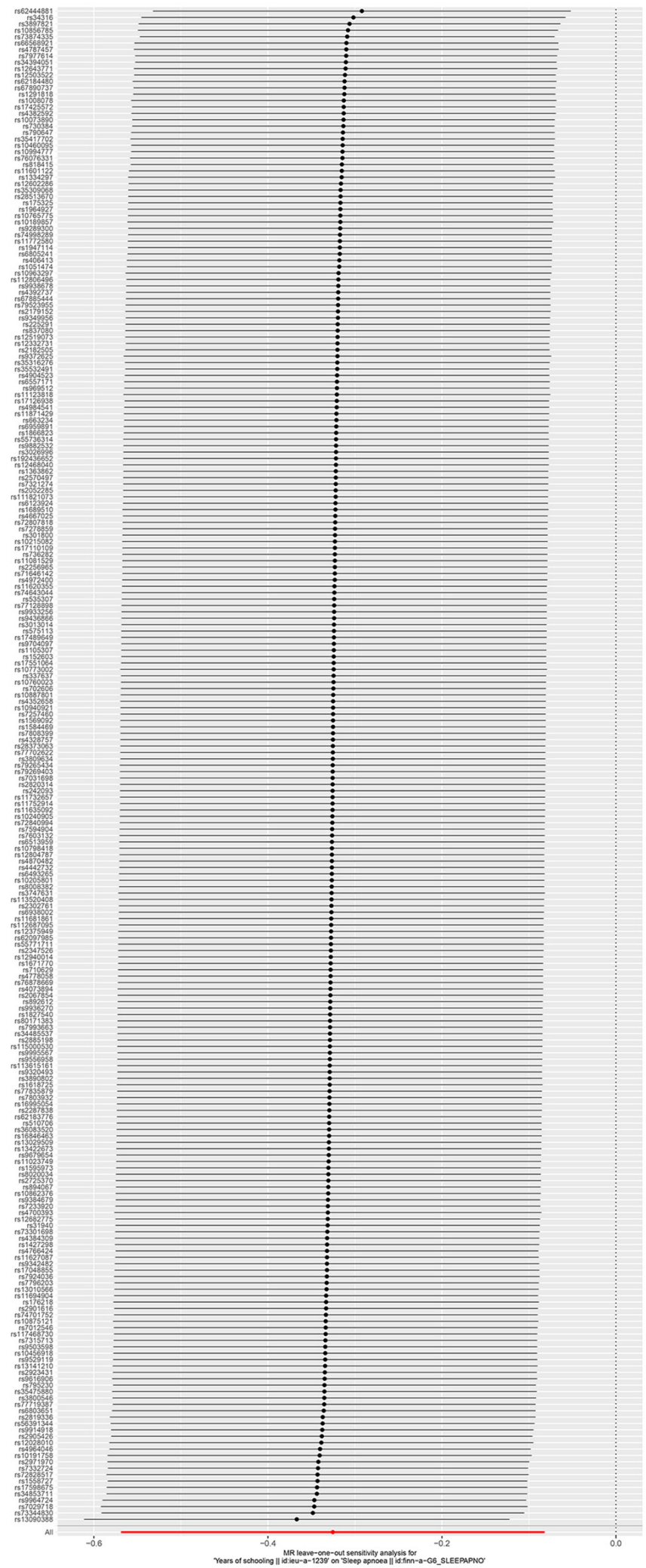

Figure S3 Leave-one-out of single nucleotide polymorphisms (SNPs) associated with educational attainment and their risk of sleep apnoea. 